\title{
Las macro-políticas educativas y el Proyecto de Educación Digital para la integración de las tecnologías desde la visión del profesorado.
}

\section{Educational macro-policies and Digital Education Project for integration of technologies from teachers' perspective.}

\author{
María José Sosa Díaz \\ Universidad de Extremadura. España \\ mjosesosa@unex.es \\ Jesús Valverde Berrocoso \\ Universidad de Extremadura. España \\ jevabe@unex.es
}

\begin{abstract}
Resumen:
Las administraciones educativas han realizado una relevante inversión económica para dotar a los centros educativos de infraestructuras digitales. No obstante, la investigación educativa demuestra que no ha habido un cambio significativo en las prácticas docentes. Las expectativas de innovación didáctica generada por la introducción de las tecnologías digitales no se han cumplido en el nivel esperado. En este artículo se analizan las «macro» y «micro» políticas educativas y sus efectos en los procesos de integración educativa de las TIC. Para ello se llevaron a cabo estudios de caso múltiples y se aplicó una metodología cualitativa-longitudinal en tres momentos diferentes. Se utilizaron técnicas de observación en el aula y entrevistas semi-estructuradas. El análisis de los datos se realizó por el método de comparación constante de la Teoría Fundamentada con apoyo en el software WebQDA. Los resultados evidencian la insuficiencia de las macro-políticas educativas para la integración de las tecnologías digitales. Es necesaria la elaboración por parte de los centros educativos de un Proyecto de Educación Digital que responda a unos objetivos pedagógicos compartidos por toda la comunidad educativa.
\end{abstract}

\section{Palabras claves:}

Tecnología Educativa, Política Educativa, Proyecto de Educación Digital, Educación Primaria.

\footnotetext{
Abstract:

The educational administration have made a relevant economic investment to equip schools with digital infrastructures. However, educational research shows that there has been no significant change in teaching practices. The expectations of pedagogical innovation generated by the introduction of digital technologies have not been fulfilled in the expected level. This article analyzes the "macro" and "micro" educational policies and their effects on the processes of educational integration of ICT. For this, case studies were carried out and a qualitative-longitudinal methodology was applied in three different moments. Observational techniques were used in the classroom as well as semi-structured interviews. The data analysis was performed by the constant comparison method of the Grounded Theory with support in WebQDA software. The results show the insufficiency of macro-educational policies for the integration of digital technologies. It is necessary the elaboration of a Project of Digital Education that responds to pedagogical objectives shared by all members of the educative community.
} 


\section{Keywords:}

Educational Technology, Education Policy, Digital Education Project, Primary Education.

\section{INTRODUCCIÓN}

La integración de las TIC en el Sistema Educativo es un proceso no exento de dificultades, cuya viabilidad está vinculada a la aplicación de políticas coherentes, a la inversión de importantes dotaciones económicas por parte de los responsables educativos o al apoyo institucional de centros y profesores, entre otros aspectos. Estos factores a menudo entorpecen una adecuada integración de las TIC en los centros, los cuales según sus características, problemáticas y necesidades se encuentran en niveles de integración tecnológica diferentes (Lázaro \& Gisbert, 2007).

Así pues, en la integración de las TIC en el Sistema Educativo confluyen múltiples factores y variables, siendo definido por numerosos académicos como un proceso complejo que evoluciona con el tiempo, en relación con dichos factores y con la participación de los actores implicados en dicho proceso (Area, 2002; De Pablos, Colás, \& González, 2010; Mioduser, Nachmias, Tubin, \& Forkosh-Baruch, 2003; Nachmias, Mioduser, Cohen, Tubin, \& Forkosh-Baruch, 2004). Además, es un proceso dinámico, en su mayoría lento, con altibajos, con avances y retrocesos, puesto que implica la transformación de patrones de comportamiento docente, los cambios en la identidad de la escuela, la mejora del rendimiento de los estudiantes y la adaptación a los cambios ambientales (Area, 2002; Nachmias et al., 2004). Por tanto, se parte de la premisa de que la integración de las TIC va más allá del mero hecho de implantar las tecnologías en el aula, o de que éstas se usen para enseñar el mismo contenido de la misma manera. Una verdadera integración de las TIC pasa por sistematizar el uso de las tecnologías en el aula, de propiciar nuevos modelos de aprendizaje y oportunidades de colaborar y construir conocimiento, a través del diseño y desarrollo de proyectos curriculares donde las TIC estén presentes (Area, 2002; Escontrela \& Stojanovi, 2004; Lázaro \& Gisbert, 2007; Protheroe, 2005).

El proceso de implantación de las TIC está determinado por las decisiones políticas que se han ido desarrollando en cada uno de los Sistemas Educativos, y que suponen el marco legal y político-social de los cambios (Colás \& Casanova, 2010; Correa \& Martínez, 2010; Nachmias et al., 2004; Sanz, Martínez, \& Pernas, 2010; Valverde, 2012; Vanderlinde, Dexter, \& Van Braak, 2012; Vanderlinde, Van Braak, \& Dexter, 2012).

Las decisiones político-educativas TIC tienen diferentes niveles: estatal, autonómica y escolar, de forma que el proceso de integración de las TIC se va delimitando y construyendo a medida que descendemos de nivel, dándole a las escuelas autonomía y una parte importante de la responsabilidad en cuanto al desarrollo del proceso (Vanderlinde, Dexter, et al., 2012; Vanderlinde, Van Braak, et al., 2012).

Por tanto, la política educativa TIC podemos analizarla, por un lado, desde una política educativa «macro», que corresponde a los niveles estatales y autonómicos, ya que está diseñada y orientada por los ministerios o consejerías de educación, y por otro, desde una política educativa «micro» desarrollada en las escuelas por profesores innovadores (Valverde, 2012).

El papel de políticas educativas en los procesos de innovación e integración de las TIC en los centros educativos públicos. María José Sosa Díaz y Jesús Valverde Berrocoso. 
1.1. MACRO POLÍticAS EDUCATIVAS TIC: Planes, PROYECTOS Y PROGRAMAS DE LAS ADMINISTRACIONES EDUCATIVAS.

En aquellos niveles macro-políticos, como es el estatal o el autonómico, se toman las decisiones para el proceso de elaboración, revisión e implementación de las medidas TIC que se llevarán a cabo en los centros educativos, además de los objetivos, el presupuesto y los efectos a conseguir con el fin de que las tecnologías formen parte de su Sistema Educativo (Correa \& Martínez, 2010; Nachmias et al., 2004; Vanderlinde, Dexter, et al., 2012).

En base a la literatura, bajo el desarrollo de las políticas educativas TIC subyacen principalmente tres razones que revelan el interés de las Administraciones Educativas por integrar las TIC en sus Sistemas Educativos (Aesaert, Vanderlinde, Tondeur, \& Van Braak, 2013; Alí, Cueva, Fernández, Murillo, \& Gómez, 2004; Area, 2002; De Pablos, Colás, \& González, 2010; Vanderlinde, Van Braak, \& Hermans, 2009; Vieira \& Silva, 2009). En primer lugar, se reconoce que el sistema escolar debe adaptarse a las nuevas características que impone la Sociedad del Conocimiento. En segundo lugar, se pretende reducir la brecha digital. Y por último, aumentar la calidad de la educación a través del uso de las TIC. Sin embargo, Valverde (2012) citando a Levin (2008) señala que los gobiernos, al crear sus políticas educativas TIC, a menudo se ven movidos por razones no propiamente pedagógicas, como por ejemplo la opinión pública, la agenda política, el tiempo, las creencias y las promesas electorales. Así pues, los poderes públicos, más preocupados por los resultados de sus acciones, realizan las medidas para la integración de las TIC con el objetivo de conseguir más votantes, basándose en la visibilidad de las disposiciones, en las creencias ciudadanas, y en que tenga resultados a corto plazo, sin tener en cuenta la opinión de los expertos y los resultados de investigación.

Las políticas educativas TIC evolucionan según las características de las sociedades, llegando a tener fases claramente diferenciadas. En este sentido, se puede dividir la evolución de las políticas educativas TIC de los países más desarrollados en tres fases.

Una primera fase sería la experimentación y grandes promesas por parte de las Instituciones Públicas, en la cual se emprenden planes, programas o proyectos a gran escala que pretenden básicamente introducir la informática e instalar algunos ordenadores en los centros educativos. A continuación, normalmente se produce un momento en el que se produce un estancamiento y desaparición de las políticas TIC desarrolladas hasta el momento (Area, 2002, 2008b).

En una segunda fase, existe un relanzamiento de los programas institucionales, aunque estas políticas educativas TIC son políticas operativas con un marcado carácter mercantilista y tecnocentrista. Las medidas desarrolladas se basan principalmente en:

a ) La financiación y adquisición de recursos para la mejora de las infraestructuras y el equipamiento, como la instalación en las aulas de ordenadores, portátiles, pizarras electrónicas, entre otros aparatos electrónicos;

b) La formación y desarrollo profesional de los docentes, caracterizada principalmente por un marcado carácter técnico y descontextualizado a las necesidades pedagógicas del profesorado (Area, 2002, 2008; Sanabria, 2006; Sosa, 2013; Valverde, 2012; Vanderlinde \& Van Braak, 2011).

El papel de políticas educativas en los procesos de innovación e integración de las TIC en los centros educativos públicos. María José Sosa Díaz y Jesús Valverde Berrocoso. 
Una última fase, que tan sólo la han promovido en algunos países más adelantados en la integración de las TIC, como es el caso del Gobierno Flamenco. Estas Instituciones Públicas han establecido programas de enseñanza oficial y obligatoria en las escuelas, estableciendo un plan de estudios con objetivos mínimos en relación con los conocimiento de las TIC, las habilidades y las actitudes para la enseñanza obligatoria (Vanderlinde \& Van Braak, 2011; Vanderlinde et al., 2009).

Se puede ver que las políticas educativas TIC evolucionan y desarrollan distintos modelos de integración de las tecnologías en el Sistema Educativo, dependiendo de la importancia que hayan ido adquiriendo éstas en la sociedad. Así pues, las primeras políticas son medidas operativas y de corte cuantitativo que se basan en un enfoque principalmente de financiación y adquisición de recursos tecnológicos. Mientras que las últimas políticas desarrollan medidas más estratégicas y cualitativas donde los aspectos claves son las condiciones psicopedagógicas del uso de las TIC y la adquisición de competencias digitales de los estudiantes (Losada, Karrera, \& Correa, 2011; Martínez, 2006; Sosa, 2013; Valverde, 2012; Vanderlinde et al., 2009).

Con todo ello, las políticas educativas TIC pretenden ser un factor determinante en el proceso de integración de las TIC en los Sistemas Educativos, potenciando el uso efectivo en el currículo escolar y contribuyendo a la transformación de la educación, a través de las medidas que se han ido implementando hasta ahora. La mejora de las infraestructuras, la alfabetización digital del alumnado y la implantación de nuevas figuras facilitadoras como el coordinador TIC y Administrador Técnico son algunas de ellas (Colás \& Casanova, 2010; De Pablos, Colás, \& Villaciervos, 2010; Losada et al., 2011). Sin embargo, las innovaciones pedagógicas y "Buenas Prácticas TIC" siguen siendo escasas y la transformación continúa siendo muy lenta. Algunos autores apuntan a las políticas educativas TIC desarrolladas por las Instituciones Públicas como factores negativos para el proceso de integración educativa de las TIC, incluso Alonso et al. (2010:72) afirma que "las innovaciones surgen, o no, en los centros y no de la política de la Administración y, en ocasiones, a pesar de ella”.

Los poderes públicos, a menudo, tratan de obligar al profesorado en el uso de las tecnologías en el aula, y fomentar así las innovaciones educativas dentro del Sistema Educativo a través de políticas de marcada orientación «arriba-abajo». Sin embargo, está demostrado que los procesos de innovación pasan por potenciar las dinámicas de «abajo-arriba», y normalmente "caen en saco roto" si las demandas de cambio son exógenas y provienen de la Administración (Losada et al., 2011; Sancho \& Correa, 2010; Sanz et al., 2010). Como señalan Sancho \& Correa (2010:20) "esta concepción de la política, al igual que los modelos de enseñanza vigentes en la mayoría de los centros de enseñanza, sitúa a los docentes como sujetos pasivos, sin biografía, sin experiencia y sin criterio, totalmente dispuestos y predispuestos para poner en práctica las ideas de otros". De esta forma, se reduce la autonomía intelectual y pedagógica del docente y, por tanto, la reflexión sobre la propia práctica educativa. En gran medida, estas políticas usurpan al profesor la toma de decisiones sobre qué herramientas tecnológicas utilizar en su aula, por qué y para qué, desmotivándole a la hora de implicarse en dichos proyectos (J. Rodríguez, 2012; Sancho \& Correa, 2010).

El profesorado a menudo ignora y desconoce elementos sustantivos de las políticas educativas TIC, lo que demuestra que estas medidas son llevadas a cabo sin tener en cuenta la opinión del cuerpo docente. Según Alonso et al. (2010), esto se debe

El papel de políticas educativas en los procesos de innovación e integración de las TIC en los centros educativos públicos. María José Sosa Díaz y Jesús Valverde Berrocoso. 
principalmente a que los instrumentos de información y relación con los centros educativos son débiles, fragmentados y poco efectivos. Mientras que, según los estudios revisados, para que una política educativa se realice con éxito, es necesario que haya contacto con los centros educativos y que los docentes participen en la elaboración de las medidas. De esta forma, las medidas serán lo más contextualizadas posibles y el profesorado se sentirá comprometido con el proceso y aprovechará al máximo los recursos que les ofrecen (Area, Sanabria, \& Vega, 2013; Vanderlinde \& Van Braak, 2011; Vanderyar, 2013).

Según la bibliografía consultada, la Administración Educativa desconoce la realidad de los centros. Se pone en evidencia la falta de apoyo institucional, pues el profesorado tiene la percepción de que la Administración es escasamente sensible a sus demandas, y frecuentemente, se ven provistos de aquello que no demandaron y no consideran prioritario (Alonso et al., 2010; García-Valcarcel \& Tejedor, 2010; Gargallo et al., 2004; Montero \& Gewerc, 2010; Vanderyar, 2013).

Esta circunstancia provoca la búsqueda de diferentes maneras de autofinanciación y autodotación de los centros educativos como estrategias alternativas de supervivencia, para adquirir aquellos recursos, materiales, infraestructuras o formación que realmente necesitan (Alonso et al., 2010).

Además, las políticas educativas TIC no reflejan los resultados de las investigaciones y recomendaciones que realizan los expertos e investigadores en el ámbito de la tecnología educativa, ya que los gobiernos consideran que no ofrecen soluciones reales a los problemas, provocando que las medidas implementadas no sean las más adecuadas para la introducción de las TIC en los centros escolares (Valverde, 2012)

\subsection{MicRo-POLÍtICAS EDUCATIVAS TIC: PlanES, PROYECTOS Y PROGRAMAS}

A partir de los niveles macro-políticos se han desarrollado medidas en cuanto a aspectos organizativos y planificación de los centros escolares. De tal forma que las políticas educativas TIC, desde un modelo de «arriba-abajo», pretenden definir la base organizativa de los centros para influir de manera determinante en la vida de las escuelas (Sancho \& Correa, 2010). Sin embargo, los centros educativos están adquiriendo cada vez más autonomía y responsabilidad, lo que hace imprescindible la existencia de una política educativa "micro" desarrollada por dichos centros, que es incluso mucho más importante y necesaria que las políticas establecidas por la Administración (Valverde, 2012; Vanderlinde, Dexter, et al., 2012). Es por ello, que este tipo de elementos dentro de la escuela han de ser tenidos en cuenta por las Instituciones Públicas para implementar políticas educativas TIC acertadas y coherentes con dichas micro-políticas educativas TIC (Tondeur, Van Keer, et al., 2008).

Numerosos estudios de investigación señalan que la política TIC de un centro educativo es un factor clave en el proceso de integración de las tecnologías. De tal modo, que la existencia de un plan, proyecto o programa institucional que impulse la innovación y el uso de las tecnologías en el centro educativo, es un paso crucial hacia su utilización integrada en los procesos de enseñanza-aprendizaje y otros procesos administrativos, y concluyen que el problema de la tecnología en las escuelas comienza con una mala planificación. Así pues, sólo aquellas escuelas que construyen una política tecnológica, tienen finalmente una exitosa integración de las TIC. (Area, 2005; Baylor \&

$\overline{\text { El papel de políticas educativas en los procesos de innovación e integración de las TIC en los centros }}$ educativos públicos. María José Sosa Díaz y Jesús Valverde Berrocoso.

Pág. 5 de 28 
Ritchie, 2002, 2002; Fishman \& Zhang, 2003; Gülbahar, 2007; Hew \& Brush, 2007; Tondeur, Van Keer, et al., 2008; Valverde, 2012; Vanderlinde et al., 2009, 2014; Vanderlinde, Dexter, et al., 2012; Vanderlinde \& Van Braak, 2010).

Autores como Valverde (2012) o Vanderlinde, Dexter, et al., (2012) denominan al documento en el que se recogen todos aquellos aspectos relativos a la política y planificación de la integración de las tecnologías en el centro, y supone la construcción de una verdadera política TIC escolar como "Plan de Política TIC" (PPT). No obstante, se considera más oportuno utilizar el concepto de "Proyecto de Educación Digital" (PED) para definir a aquel documento que recoge tanto la visión como la misión, objetivos, expectativas, metas, contenidos o acciones para implementar las TIC en dicho centro escolar, además de las especificaciones técnicas y de infraestructura existentes y futuras del centro (Vanderlinde et al., 2008; Vanderlinde, Dexter, et al., 2012). Así pues, este documento debe ofrecer elementos tanto "estratégicos" como "operativos" que guíen el proceso de integración de las TIC en el centro educativo (Vanderlinde, Van Braak, et al., 2012; Vanderlinde, Van Braak, \& Tondeur, 2010).

Sin embargo, no se puede olvidar que el "Proyecto de Educación Digital", más que una secuencia de actividades que la escuela espera lograr, también debe proporcionar la filosofía latente en el centro educativo respecto al binomio tecnologíaeducación. El PED debe procurar al centro una política polifacética fruto de la cultura y el clima escolar que se produce en la comunidad educativa respecto a las tecnologías (Baylor \& Ritchie, 2002; Vanderlinde et al., 2008; Vanderlinde, Van Braak, et al., 2012; Vanderlinde et al., 2010). Para ello, aunque el documento sea escrito por una persona, que puede ser el coordinador TIC, el proceso de creación del contenido del documento debe ser un trabajo colaborativo donde participe toda la comunidad educativa.

En esta línea, las investigaciones apuntan a que cualquier planificación educativa tiene mayores posibilidades de llevarse a cabo si ha participado conjuntamente todo el claustro en la toma de decisiones y la construcción de la visión compartida del papel de las TIC. Por tanto, un buen indicador del uso de las TIC es el compromiso y la participación del profesorado en la creación del Proyecto de Educación Digital, ya que este debe ser un proceso creado en equipo, con visión estratégica y de futuro (Fishman \& Zhang, 2003; Hadjithoma \& Karagiorgi, 2009; Sánchez et al., 2011; Tondeur, Van Keer, et al., 2008; Vanderlinde, Dexter, et al., 2012; Vanderlinde, Van Braak, et al., 2012; Vanderlinde \& Van Braak, 2010; Vanderlinde et al., 2008, 2010).

Asimismo, el compromiso docente es un aspecto importante que se debe valorar. Si los docentes están convencidos de la necesidad de integrar las TIC en el centro y de conformar una fuerte visión, es menos probable que la implementación del proyecto sea abandonado (Kopcha, 2012). La labor de los líderes de los centros educativos es también un factor determinante en el proceso de creación de "Proyecto Educativo Digital". La literatura científica enfatiza la importancia del liderazgo del equipo directivo como elemento potenciador, motor de innovación y cambio dentro de la escuela, y las funciones del coordinador TIC, como dinamizador y orientador del proceso (Aguaded, Tirado, \& Cabero, 2008; Espuny, Gisbert, \& Coiduras, 2010; Vanderlinde et al., 2014; Vanderlinde, Dexter, et al., 2012; Vanderlinde, Van Braak, et al., 2012).

En el proceso de creación de desarrollo del proyecto también debe estar implicada la Administración, con una política de acompañamiento y apoyo institucional a los

El papel de políticas educativas en los procesos de innovación e integración de las TIC en los centros educativos públicos. María José Sosa Díaz y Jesús Valverde Berrocoso.

Pág. 6 de 28 
centros. Así pues, los poderes públicos deberían potenciar la creación de los planes tecnológicos en los centros, evaluar con criterios sólidos los proyectos, y prestar los servicios de asesoramiento necesarios a los docentes, coordinadores TIC, equipos directivos y personal administrativos para que superen los posibles obstáculos (Aguaded et al., 2008; Gülbahar, 2007; Lázaro \& Gisbert, 2007; Pérez, Aguaded, \& Fandos, 2009).

En cualquier caso, el proceso de creación del "Proyecto Educativo Digital" constituye una visión compartida sobre el papel que ocupan las TIC en la educación (Hew \& Brush, 2007), los objetivos que se pretenden con ellas, los métodos y materiales que pueden utilizarse para mejorar el aprendizaje, así como los puntos fuertes y débiles respecto a la organización de las TIC en el centro, lo que supondrán la elaboración de las medidas adecuadas para conseguir la integración de las TIC de forma efectiva (Espuny et al., 2010). El proceso de creación de dichos aspectos supone una oportunidad para el claustro de desarrollar un proceso de reflexión sobre la propia práctica educativa y sus creencias en relación a las TIC que, sin duda, mejora la introducción de las tecnologías en el aula (Aguaded et al., 2008; Vanderlinde \& Van Braak, 2011; Vanderlinde et al., 2009, 2010). Incluso algunos autores señalan que es más importante el proceso de elaboración del "Proyecto Educativo Digital", debido al proceso de reflexión que se produce durante la creación, que el mismo documento en sí (Gülbahar, 2007; Vanderlinde et al., 2010).

En todo caso, para una adecuada incorporación de las TIC en el centro y construir una visión compartida de los docentes, el "Proyecto Educativo Digital" debe proporcionar un modelo de explotación didáctica de las tecnologías digitales. El documento debe recoger elementos que guiarán el proceso de integración escolar de la tecnología en el centro educativo.

No obstante, la realidad es que la mayoría de las escuelas no cuentan con un "Proyecto Educativo Digital" que implique, al menos, alguna visión sobre el uso de las TIC o modificación de las prácticas didácticas dominantes (Tilve \& Álvarez, 2009; Tilve, Gewerc, \& Álvarez, 2009; Vanderlinde, Dexter, et al., 2012). Algunas razones por las que las escuelas no tienen este tipo de elemento tan importante en el proceso de integración de las tecnologías son, fundamentalmente, la falta de tiempo durante el ejercicio profesional tanto en docentes como equipos directivos y la ausencia de presión y apoyo gubernamental (Vanderlinde, Dexter, et al., 2012). En aquellos centros educativos donde finalmente se confecciona este tipo de planificación, el trabajo lo realiza principalmente sólo aquel profesor "amante de las tecnologías", basándose en el autoaprendizaje y realizando un gran esfuerzo personal, con resultados académicos de los que no existen suficientes evidencias científicas. Normalmente, el plan no es comunicado ni compartido con la toda la comunidad educativa, y tiene escasa valoración por parte de la Administración, que no ofrece suficiente apoyo técnico (Valverde, 2012). Además, no todos los centros educativos al construir el "Proyecto Educativo Digital" tienen en cuenta todos y cada uno de los aspectos señalados, sino que cada uno realiza un tratamiento diferente del proyecto, que responde a la idiosincrasia del propio centro y, sobre todo, al interés del claustro y del equipo directivo hacia las TIC (Sánchez et al., 2011).

$\overline{\text { El papel de políticas educativas en los procesos de innovación e integración de las TIC en los centros }}$ educativos públicos. María José Sosa Díaz y Jesús Valverde Berrocoso. 


\section{METODOLOGÍA DE INVESTIGACIÓN}

\subsection{OBJETIVOS}

La intención a lo largo de la investigación ha sido profundizar en la integración de las TIC en los centros educativos de primaria e infantil, de tal forma que se pueda conocer el estado actual de este proceso y los fenómenos que se están produciendo alrededor del mismo. Para ello se pretende explorar y analizar el modelo político tanto a nivel macro como micro que están influyendo de manera determinante en los procesos de innovación y de la integración de las TIC.

Según las características del problema de investigación que nos ocupa y los objetivos planteados, se ha seleccionado llevar a cabo un estudio de caso múltiple desde una metodología primordialmente de corte cualitativo como es la Teoría Fundamentada. Ya que sobretodo interesa comprender e interpretar desde un análisis comprensivo, holístico y profundo este fenómeno (Strauss \& Corbin, 2002, p. 12). En este sentido, se prefiere utilizar estrategias de observación y entrevistas abiertas con las cuales se renuncia a la búsqueda de relaciones causales propias de metodologías cuantitativas.

\subsection{DISEÑO DE LA INVESTIGACIÓN}

En cuanto a la dimensión temporal, se ha desarrollado una investigación longitudinal, dentro de la cual se han llevado a cabo tres tipos de Estudios de Casos, dependiendo del momento y la fase en la que nos encontramos. Así pues como se puede observar en la tabla 1, en el primer estudio de investigación se llevó a cabo un estudio colectivo intrínseco. Se realiza el estudio de cuatro centros educativos de primaria, con el objetivo de indagar sobre las especificidades y aspectos que caracterizan a estos centros en cuanto a las TIC se refiere. En un segundo Estudio de Casos se desarrolla un estudio intrínseco de un determinado caso, puesto que se consideró necesario indagar en las características organizativas que este centro desarrollaba en el estudio de campo anterior, por lo que se decidió estudiar más pormenorizadamente este tipo de aspectos. Y por último, en el tercer estudio de investigación, se implementa un estudio colectivo extrínseco de los cuatro centros educativos que anteriormente se habían estudiado, con el objetivo de formular generalizaciones y teorías.

Tabla 1: Tipos de Estudios de Casos desarrollados

\begin{tabular}{llll} 
Fase & Tipo de caso & Participación & Objetivo \\
\hline 1 Estudio & Estudio colectivo intrínseco & 4 CEIP & $\begin{array}{l}\text { - Identificar y comprender todos los } \\
\text { aspectos TIC }\end{array}$ \\
\hline 2 Estudio & Estudio intrínseco & 1 CEIP & - Indagar en un aspecto organizativo \\
\hline 3 Estudio & Estudio colectivo extrínseco & 4 CEIP & - Generalizar y teorizar \\
\hline
\end{tabular}

\subsection{INSTRUMENTO DE RECOGIDA DE INFORMACIÓN}

Para la recolección de información de los distintos miembros de la Comunidad Educativa se usará la entrevista semi-estructurada acompañada de un guión de preguntas abiertas. Así pues, en el desarrollo de los tres estudios de investigación que han formado parte de nuestro trabajo la entrevista se desarrolla a partir de cuestiones que persiguen reconstruir lo que para él -el entrevistado- significa el problema de estudio (Rodríguez,

El papel de políticas educativas en los procesos de innovación e integración de las TIC en los centros educativos públicos. María José Sosa Díaz y Jesús Valverde Berrocoso. 
Gil, \& García, 1999, p. 168). Los guiones de las entrevistas elaborados, tienen una estructura predeterminada que el investigador ha elaborado con anterioridad a la reunión. No obstante, esta estructura pretende ser flexible y modificable según el desarrollo de la entrevista y en función de las respuestas dadas, en línea de la información que se desea conocer.

Para elaborar el protocolo de las entrevistas se ha tenido presente las cuestiones o aspectos a profundizar según se identificaron en la primera revisión bibliográfica. Sin embargo, los siguientes protocolos de entrevistas responden a la necesidad de indagación que el investigador determina al obtener los resultados de estudio anterior.

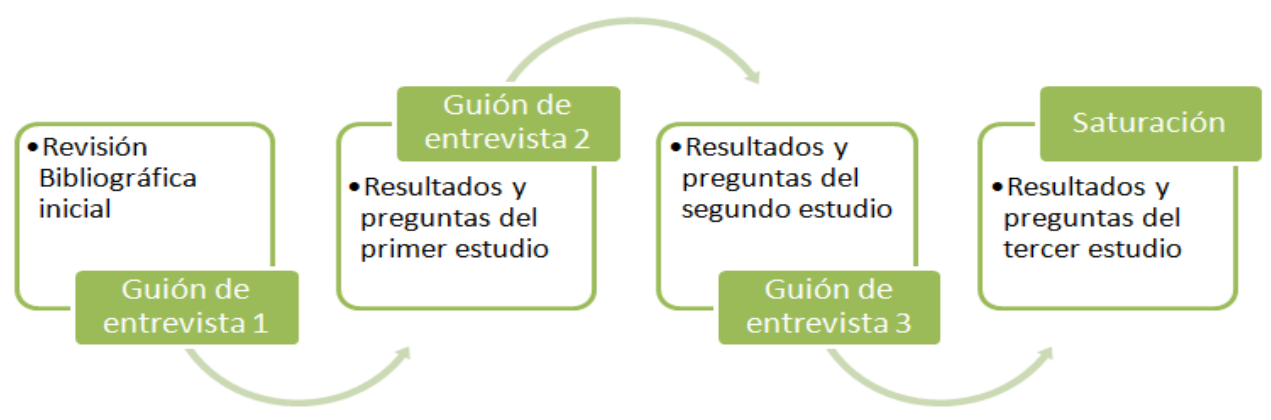

Ilustración 1: Proceso para la elaboración del guión de entrevistas

\subsection{PARTicipantes}

A lo largo de la investigación han participado 57 personas entre coordinadores TIC, profesorado, miembros de equipos directivos, familias y asesores TIC. A continuación, se presenta en la tabla 2 la totalidad de entrevistas individuales que se realizaron a lo largo de los tres estudios de investigación. En ella se señala el rol que cumple el entrevistado, así como la etapa educativa a la que pertenece y el código que se le ha asignado.

El papel de políticas educativas en los procesos de innovación e integración de las TIC en los centros educativos públicos. María José Sosa Díaz y Jesús Valverde Berrocoso. 
Tabla 2: Resumen de participantes y códigos de confidencialidad

\begin{tabular}{|c|c|c|c|c|c|}
\hline Estudio & Subunidad & CÓDIGOS & & & \\
\hline & Centro & CP_01 & CP_02 & CP_03 & CP_04 \\
\hline \multirow{5}{*}{$\begin{array}{l}\text { Primer } \\
\text { Estudio } \\
\text { (E1) }\end{array}$} & AMPA & MP01 & MPO2 & MP03 & MP04 \\
\hline & Coord. TIC & $\mathrm{C} 01$ & $\mathrm{C} 02$ & $\mathrm{C} 03$ & $\mathrm{C} 04$ \\
\hline & $\begin{array}{l}\text { Eq. } \\
\text { Directivo }\end{array}$ & ED01 & ED02 & ED03 & ED04 \\
\hline & Estudiantes & ES01_1C ES01_3C & ES02_2C & ES03_Ap & ES04_2C \\
\hline & Profesores & P01, P02 & P03, P04 & $\begin{array}{l}\text { P05-P06, P07, } \\
\text { P08 }\end{array}$ & $\begin{array}{l}\text { P09, P10, P11, } \\
\text { P12, P13, P14 }\end{array}$ \\
\hline \multirow[t]{5}{*}{$\begin{array}{l}\text { Segundo } \\
\text { estudio } \\
\text { (E2) }\end{array}$} & AMPA & $\begin{array}{l}\text { PreAMPA, } \\
\text { SecAMPA, } \\
\text { AMPA1, AMPA2, } \\
\text { AMPA3, }\end{array}$ & & & \\
\hline & $\begin{array}{l}\text { Asesores } \\
\text { TIC }\end{array}$ & $\begin{array}{l}\text { TecCPR, AsCPR1, } \\
\text { AsCPR2 }\end{array}$ & & & \\
\hline & Coord. TIC & CoorTIC & & & \\
\hline & $\begin{array}{l}\text { Eq. } \\
\text { Directivo }\end{array}$ & $\begin{array}{l}\text { DirEQD, JefeEQD, } \\
\text { SecEQD }\end{array}$ & & & \\
\hline & Profesores & $\begin{array}{l}\text { ProfINF1,ProfINF2 } \\
\text {, ProfINF3, } \\
\text { ProfPRI1, } \\
\text { ProfPRI2, } \\
\text { ProfPRI3, }\end{array}$ & & & \\
\hline \multirow{2}{*}{$\begin{array}{l}\text { Tercer } \\
\text { estudio } \\
\text { (E3) }\end{array}$} & Coord. TIC & Dir_cp01 & Dir_cp02 & Dir_cp03 & Dir_cp04 \\
\hline & $\begin{array}{l}\text { Eq. } \\
\text { Directivo }\end{array}$ & Coor_cp01 & Coor_cp02 & Coor_cp03 & Coor_cp04 \\
\hline
\end{tabular}

\subsection{ANÁlisis DE DATOS}

La Teoría Fundamentada (GroundedTheory), ha sido la clave para interpretar los resultados cualitativos de la presente investigación puesto que hace explícitos los procedimientos de análisis cualitativo con el propósito de ir generando la teoría de forma sistemática (Glaser \& Strauss, 1967; Strauss \& Corbin, 2002). Como se muestra en la ilustración 2, en la presente investigación se ha seguido el procedimiento de comparación constante que según Glaser y Straus (1967) dividen en cuatro etapas:

$1^{\circ}$ Codificación abierta, comparación de los datos: El proceso de categorización y comparación se ha realizado en matrices resumen.

$2^{\circ}$ Codificación axial, integración de cada categoría con sus propiedades: El proceso de síntesis y selección de la información importante se ve reflejado en la creación de memorandos en los que se puntualizan las primeras impresiones de los datos recogidos.

El papel de políticas educativas en los procesos de innovación e integración de las TIC en los centros educativos públicos. María José Sosa Díaz y Jesús Valverde Berrocoso. 
$3^{\circ}$ Codificación selectiva, delimitar la teoría que comienza a desarrollarse: Este proceso se ha realizado a través de la construcción de un esquema emergente que narra las relaciones entre las categorías describiendo el proceso o fenómeno.

$4^{\text {o }}$ Saturación teórica de los incidentes propios de cada categoría: Se da por concluida la recogida de datos, al no surgir nuevas ideas y relaciones que amplíen la investigación, por lo que se procede a describir los resultados del presente trabajo.

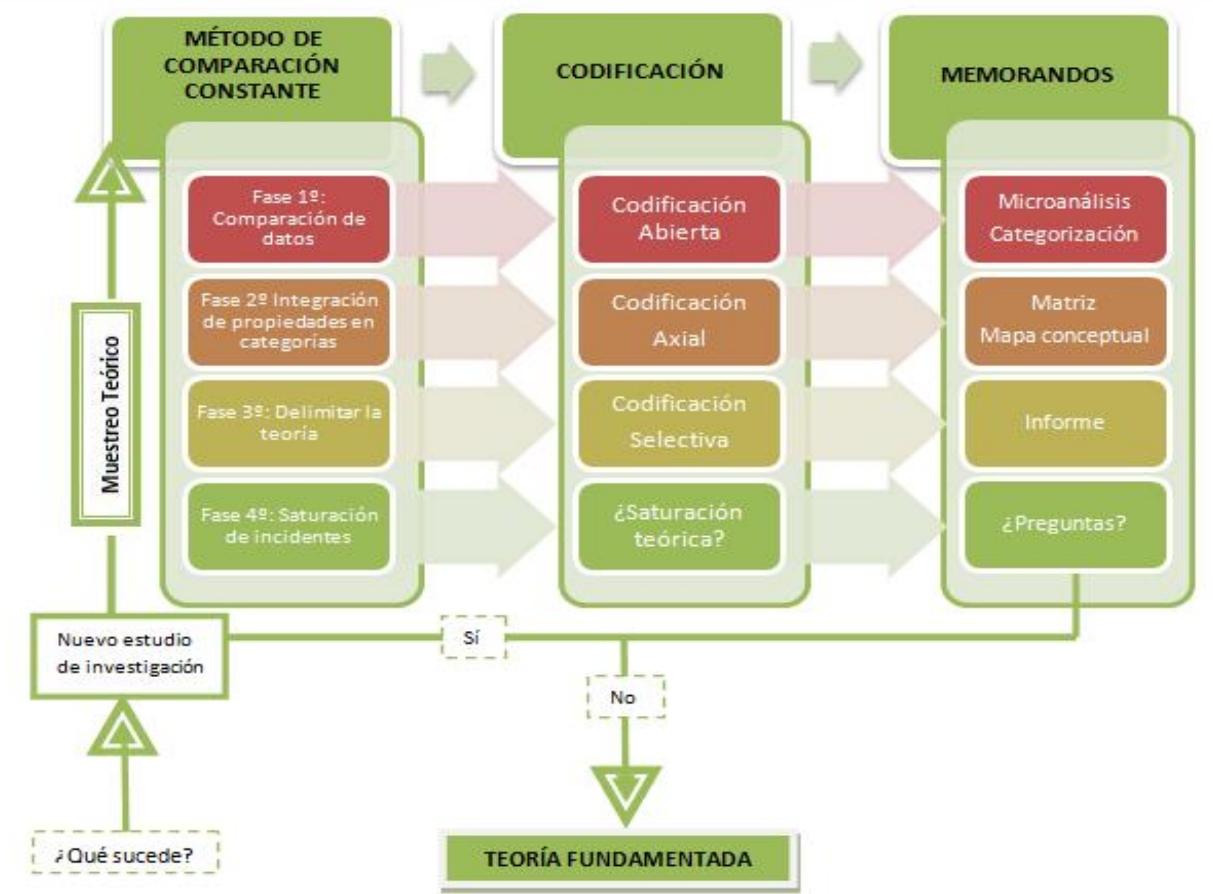

Ilustración 2: Proceso de análisis a través de la Teoría Fundamentada (elaboración propia)

\section{RESULTADOS}

La Administración Educativa es considerada por la mayor parte de los entrevistados como la máxima responsable de la integración de las TIC en los centros educativos, ya que adopta las decisiones relacionadas con la inversión o con las líneas generales de política educativa. En este sentido, a continuación se realiza un análisis de la percepción que tiene la comunidad educativa sobre la política educativa desarrollada por la Administración, así como consideraciones de mejoras.

\subsection{VALORACIÓN DE LA COMUNIDAD EDUCATIVA DE LOS DISTINTOS ASPECTOS DE LA POLÍ́TICA TIC}

La política TIC llevada a cabo por la Administración Educativa de Extremadura, es considerada por toda la comunidad educativa en los tres estudios de investigación como una gran apuesta para mejorar de la calidad de la enseñanza (P02, referencia 1, ADMINTIC, E1; P12, referencia 1, ADMINTIC, E1; Coor_cp03, referencia 1, ADMINTIC, E3). Muchos entrevistados valoran el gran esfuerzo económico, instalando, en todos los centros de educación primaria e infantil, aulas con dispositivos digitales.

El papel de políticas educativas en los procesos de innovación e integración de las TIC en los centros educativos públicos. María José Sosa Díaz y Jesús Valverde Berrocoso. 
"Yo creo que la Junta de Extremadura está invirtiendo bastante en Educación y sobre todo en nuevas tecnologías. [...] La Junta de Extremadura yo creo que está haciendo unos esfuerzos bastantes grandes en Educación [...], y está dotando bien a los centros y está creando los cursos necesarios para que los maestros pues podamos llevar a cabo esas Nuevas tecnologías, yo creo que sí". (P03, referencia 1, ADMINTIC, E1)

Sin embargo, aunque la visión de la comunidad educativa es positiva en cuanto a la política TIC, los entrevistados se encuentran descontentos debido principalmente a la forma en la que se está desarrollando. Una inmensa mayoría coinciden en que el modelo no ha sido el adecuado, y que se han cometido errores, motivos por los que no se ha producido una integración real de las TIC (Dir_cp01, referencia 1, ADMINTIC, E3).

\section{a) No se ha valorado las necesidades reales de los centros educativos}

La instalación de los equipos informáticos en los centros educativos se ha realizado de manera abrupta, viéndose los docentes desbordados por la implantación de recursos que a menudo no sabían utilizar (ED04, referencia 1, ADMINTIC, E1). Los entrevistados consideran que se ha construido "la casa por el tejado", expresión que utilizan para explicar que la introducción de las TIC en el Sistema Educativo Extremeño vino por la imposición de la Administración Educativa, y sin valorar los intereses y necesidades reales de la escuela y sus docentes (Dir_cp02, referencia 2, ADMINTIC, E3). De esta forma, la Administración ha instalado los mismos recursos tecnológicos y humanos independientemente de las características de los centros educativos y el interés de su claustro (P13, referencia 6, ADMINTIC, E1). Hecho que provoca el desinterés de los docentes ante el desarrollo de esta política TIC, quedando infrautilizados, obsoletos e inoperativos los recursos tecnológicos instalados.

"Supongo que ya lo habréis oído muchas veces, que se empezó la casa por el tejado, y se mandaron ordenadores porque tocaba mandar ordenadores, pero nadie estaba preparado, ni los centros tenían lugares, porque nosotros tenemos ahí un 'micro aula' ni siquiera un 'miniaula' donde se metieron veinte ordenadores, pero no caben veinte alumnos, caben pero no pueden manejarse, entonces ese tipo de cosas, entonces falto un estudio de centro a centro y faltó preguntar a cada centro que es lo que realmente necesitarían". (Coor_cp02, referencia 2, E3)

\section{b) Los recursos tecnológicos son insuficiente e inservibles}

Los equipos informáticos, que la Administración ha instalado en los centros, en numerosas ocasiones no son actualizados correctamente (Dir_cp03, referencia 1, ADMINTIC, E3). Según los entrevistados está provocando que aquellos docentes interesados y motivados por el uso de las TIC en el aula, tengan dificultades para desarrollar su docencia con las tecnologías.

"Tanto material muchas veces es inservible porque..., no sé, yo exactamente no sé cuántos ordenadores aquí, hay aquí en, en el colegio, no sé 40 y tantos, 50 y tantos, puede haber. Creo que a lo mejor si hubieran mandado la mitad y hubieran sido mejores, o hubieran buscado otras empresas, o no sé, es que hay hay un montón de historias y he estado en equipos directivos anteriormente, entonces...". (P09, referencia 2, ADMINTIC, E1)

\footnotetext{
$\overline{\text { El papel de políticas educativas en los procesos de innovación e integración de las TIC en los centros }}$
} educativos públicos. María José Sosa Díaz y Jesús Valverde Berrocoso. 
Este problema se agrava en el momento en el que la Administración no pone a disposición de los centros escolares suficiente personal técnico que solucionen los problemas que, a menudo, se encuentran los docentes (P14-15, referencia 2, ADMINTIC, E1). Mientras tanto, los centros educativos deben seguir buscando alternativas para reparar aquellas averías más urgentes recurriendo principalmente a coordinadores TIC o los docentes con más conocimientos informáticos (P13, referencia 1-2, ADMINTIC, E1). Incluso, en ocasiones, intentan solventar las carencias de sus infraestructuras TIC, utilizando distintas formas de financiación como acuerdos con editoriales o fondos económicos obtenidos por premios. Sin embargo, la Administración Educativa es bastante inflexible en esta cuestión y es la única que tiene potestad para comprar, subvencionar o reparar los equipos informáticos que se utilizan en el centro educativo, lo que dificulta la realización de dichas medidas alternativas (SecEQD, referencia 1, PROADMIN, E2).

En este sentido, los profesores entrevistados en los dos últimos estudios de investigación, señalan que la Administración Educativa tiene parcialmente abandonado a la etapa educativa de primaria e infantil respecto a la integración de las TIC (Dir_cp01, referencia 1, ADMINTIC, E3; Dir_cp03, referencia 1, ADMINTIC, E3). Durante los años de desarrollo de los distintos programas TIC, no sólo ha instalado menos recursos tecnológicos que en la etapa de Educación Secundaria, sino que le ha dado menos cobertura técnica y no ha evaluado las necesidades de cada uno de los centros educativos para atender sus demandas.

"Pero no podemos estar así. Somos... en esta movida tan maravillosa que se ha montado con las Nuevas tecnologías, que me encantan, que bueno... la han montado para secundaria, tienen un administrador informático para cada instituto, nosotros compartimos un administrador informático para toda la demarcación del CPR. Centros no sé cuando serán, lo iba a mirar el otro día..., que no nos lo pongan, que no nos pongan los ordenadores, que nos digan 'Esta experiencia, este proyecto maravilloso con la que vamos a salir en todas las televisiones del mundo es para secundaria y primaria no lo tiene'. Pues seguimos con nuestro lápiz y nuestro cuaderno y genial, pero ponen la miel en la boca, y luego pues ala". (CoorTIC, referencia 1, ADMINTIC, E2)

\section{c) La formación del profesorado ha sido deficiente}

Además, como se evidencia en el comentario anterior, la Administración Educativa no ha favorecido una adaptación adecuada del profesorado a las tecnologías (Dir_cp03, referencia 1, ADMINTIC, E3). Los docentes afirman que no se han establecido las medidas de motivación, ni la formación necesaria, antes de la instalación de los equipos informáticos y, sobre todo, antes de la instalación del sistema operativo LinEx (Coor_cp03, referencia 1, ADMINTIC, E3).

"Personalmente te voy a decir, a mí me costó tanto que es que creo que los cursos que dieron al principio poquitos y madre mía, difícil de asumir [...]. Creo que debían de hacer sido, más escalonados, más tiempo. No te pueden venir de golpe y porrazo, de la noche a la mañana, plantarte un montón de ordenadores, este nuevo sistema y mañana vengo te doy dos cursos de 8 horas o en una semana”. (P05-P06, referencia 1, ADMINTIC, E1)

El papel de políticas educativas en los procesos de innovación e integración de las TIC en los centros educativos públicos. María José Sosa Díaz y Jesús Valverde Berrocoso. 


\section{d) No ha habido seguimiento del proceso de integración de los recursos}

Asimismo, la política TIC de la Administración no sólo tiene deficiencias en cuanto al modelo inicial de implantación de las tecnologías dentro de los centros, sino que el proceso de seguimiento también es deficiente (ED01, referencia 3, ADMINTIC, E1). Los docentes manifiestan que la Administración Educativa no ha realizado una valoración de las necesidades una vez que han sido instalados los dispositivos digitales en los centros. Además, no se ha comprometido a motivar y a apoyar a los docentes mediante compensaciones económicas o formación adaptada a sus necesidades de forma continua (P07, referencia 1, PROADMIN, E1).

"Entonces claro, mantenimiento, seguimiento, por ejemplo de la Junta, y como lo lleváis, que necesitáis, pues eso no lo veo. Es decir, nos pone la pizarra digital, bueno necesitáis algún apoyo más, alguna formación más de esto y búscate la vida". (C01, referencia 1, ADMINTIC, E1)

\section{f) La política educativa TIC supone un marketing político a la Administración}

Por todo ello, una gran parte de los entrevistados de los tres estudios de investigación afirman que la política educativa TIC de la Administración Educativa de la Comunidad Autónoma de Extremadura se ha preocupado principalmente de la instalación masiva de los ordenadores y pizarras digitales. Se ha dejado en un segundo plano las otras líneas de implantación TIC como la formación docente, los recursos humanos con perfil técnico y la creación de materiales didácticos interactivos (MP01, referencia 4, ADMINTIC, E1). En este sentido, buena parte de los entrevistados sostienen que la política TIC extremeña obedece a intereses políticos y económicos (Coor_cp03, referencia 1, ADMINTIC, E3). Se considera entonces que la Administración Educativa sólo establece la política educativa basada en TIC con un objetivo de marketing político, que seducirá a la ciudadanía cuando perciba que en todos los centros educativos tienen equipos informáticos (ProfPRI3, referencia 2, PROADMIN, E2).

"Ya te lo he dicho un poco antes. El poco apoyo que tenemos, porque no dan suficientes informáticos para cubrir esas carencias que a veces tenemos, ¿no?, pero bueno yo la verdad que a veces me lo planteo si las cosas se hacen por un interés político exclusivamente, ¿no?”. (C01, referencia 2, ADMINTIC, E1)

\subsection{LA COMUNIDAD EDUCATIVA EXIGE UN NUEVO MODELO DE POLÍTICA TIC}

Por tanto, según las deficiencias encontradas por parte de la comunidad educativa en cuanto al proceso de introducción de las TIC en los centros escolares, se considera que la Administración debe rectificar, y crear un nuevo modelo de integración de las TIC aplicado bajo una serie de principios básicos.

\section{a) Mejora de la dotación de recursos tecnológicos}

Por un lado, los docentes demandan más equipos informáticos y pizarras digitales en las aulas ordinarias y un mejor acceso a Internet, acompañado de más recursos humanos para el apoyo técnico de instalación y mantenimiento de los equipamientos (Coor_cp01, referencia 2, PROADMINTIC, E3; ED03, referencia 1, PROADMIN, E1; P14-P15, referencia 3, PROADMIN, E1). Sólo de esta forma los recursos tecnológicos

El papel de políticas educativas en los procesos de innovación e integración de las TIC en los centros educativos públicos. María José Sosa Díaz y Jesús Valverde Berrocoso.

Pág. 14 de 28 
tendrán un buen funcionamiento en cada momento y serán utilizados por los profesores en su práctica docente, sin preocuparse por los problemas técnicos que puedan acontecerles.

"Nada más que sería interesante pues eso que mejoraran los equipos de los que disponemos, sin duda, que fueran más nuevos, que fueran más rápido, que el acceso a Internet fuera también, hubiera más banda ancha”. (P12, referencia 3, PROADMIN, E1)

El modelo que consideran más adecuado es el de una Pizarra Digital por aula, un aula de informática, conexión a Internet y suficientes administradores técnicos. No se considera que el modelo 1 a 1 , sea adecuado para la educación primaria y consideran que no sería efectivo.

"No se trata de traer a los centro un ordenador por alumno, que me parece totalmente ineficaz o un portátil por alumno que me parece totalmente ineficaz, y sí que sería más conveniente tener una pizarra por aula, Internet que funcione en condiciones, y algún tipo de técnico que por lo menos supervise cada 5 o 6 centros pues como funcionan y como se puede solucionar o solventar cualquier tipo de problema que haya". (Dir_cp04, referencia 1, ADMINTIC, E3)

En este nuevo modelo es necesario realizar una valoración de los recursos tecnológicos de los centros, de las dificultades e intereses de los docentes o los centros educativos, y a partir de las conclusiones proponer unas medidas de actuación para integrar las TIC de forma efectiva. De esta forma, se contaría con la complicidad de los docentes, se valoraría su opinión y se ajustarían las medidas a la realidad educativa.

"Lo primero sería escuchar a los profesores, pero no escuchar a una parte de los profesores, pues si escucha a los coordinadores TIC van a recibir un tipo de contestación aunque los coordinadores TIC en cierta manera van a trasladar la evidencia de su centro, y las opiniones de su centro y va estar siempre sesgado porque al fin y al cabo el coordinador TIC sería el más adelantado del centro, escuchar a todos los profesores, y escuchar centro por centro, y dar la autonomía en cada centro de que decida qué proyecto tecnológico quiere y a partir de ahí dar el presupuesto para el proyecto completo o medio proyecto o lo que sea". (Coor_cp04, referencia 1, PROADMINTIC, E3)

Para ello se considera más correcto llevar a cabo convocatorias de centros pilotos que soliciten recibir los recursos tecnológicos que consideren oportunos. Ya que esta iniciativa partiría del interés del profesorado, se rentabilizarían los recursos tecnológicos y se motivaría a otros centros y docentes a integrar las TIC (Coor_cp02, referencia 2, PROADMINTIC, E3). Solicitando, además, a la Administración tener más independencia y autonomía económica para la compra de recursos tecnológicos o para la contratación de personal informático, así como para poder intercambiar recursos tecnológicos que estén en estado óptimo que otros centros no utilicen y puedan ser donados o intercambiados.

"Presentarnos nuestro proyecto de lo que queréis hacer y vamos a ver con que presupuesto optar, y encargaros vosotros de comprar lo que haga falta, por ahí debería seguir, en cada centro, si aquí necesitamos cinco pizarras, podernos comprar cinco pizarras y si nos sobra ordenadores pues

El papel de políticas educativas en los procesos de innovación e integración de las TIC en los centros educativos públicos. María José Sosa Díaz y Jesús Valverde Berrocoso. 
a lo mejor en otro colegio necesitan ordenadores, pues que se los lleven o intercambiamos como los cromos o lo que sea, faltó eso, yo creo que la Administración va por un lado, y a nosotros nos llega las cosas, y con lo que nos llegan intentamos hacer lo mejor posible”. (Coor_cp04, referencia 1, ADMINTIC, E3)

\section{b) Es necesario más apoyo al profesorado}

Y por otro lado, apoyar al profesorado mediante programas obligatorios de formación que sean de calidad y que estén en manos de especialistas en Tecnología Educativa (C02, referencia 1-2, PROADMIN, E1). Además, se advierte que es necesario compensaciones económicas por el uso didáctico de las TIC y más tiempo a los docentes para formarse (Dir_cp02, referencia 2, PROADMIN, E3). Según los entrevistados es imprescindible que además la Administración informe y explique las decisiones políticas relacionadas con las TIC que afectan al profesorado (P14-P15, referencia 4, PROADMIN, E1). De esta forma se reducirá el sentimiento de incertidumbre entre el profesorado, puesto que conocerán los cambios específicos que sufrirá el Sistema Educativo y cómo afectarán a su práctica docente, se podrán anticipar a algunos problemas y adaptarse a la nueva situación que está por venir.

"Entonces claro si la Junta nos diera más información, se evitaba eso, se rentabilizaba más, la gente estaría más al tanto con este tema, lo que sí es verdad. [...] El profesor necesita muchísimo tiempo para buscar aplicaciones que puedan llevar a cabo los chavales de forma semiautónoma. [...] Entonces que te formen en este aspecto suficiente". (C01, referencia 1-2, PROADMIN, E1)

\section{c) Apoyo a las familias}

Por último, es también relevante tener en cuenta la importancia de la implicación de las familias en el proceso de integración de las TIC en los centros educativos. Es por ello, que los entrevistados señalan que la Administración debe ofrecer ayudas para la formación en competencias digitales a los padres y madres, así como para la adquisición de equipos informáticos o para acceder a Internet en los hogares.

"La Administración tendría que encargarse a través del centro, si quiere, y con profesionales externos a él, en formar a los padres en muchos aspectos". (Dir_cp02, referencia 1, PROADMIN, E3)

Así pues, se puede concluir que las características de la política educativa TIC, y por tanto, el modo de implantación de las tecnologías en los centros, es un factor muy importante a la hora de conseguir una adecuada integración de las tecnologías e influirá notablemente en la implicación del profesorado en el uso didáctico de las tecnologías dentro del aula (Coor_cp03, referencia 1, RESAE, E3).

Siendo así necesaria, en cualquier Política TIC, además de instalar equipos informáticos de forma escalonada (Dir_cp02, referencia 1, PROADMINTIC, E3), valorar las necesidades y características de los centros y los docentes (Dir_cp01, referencia 1, RESAE, E3), dar suficiente apoyo y formación al profesorado y a las familias (Dir_cp01, referencia 1, PROADMINTIC, E3), así como darle un adecuado seguimiento del proceso de instauración de las TIC y mantenimiento de todos los recursos tecnológicos (Dir_cp02, referencia 2, RESAE, E3).

El papel de políticas educativas en los procesos de innovación e integración de las TIC en los centros educativos públicos. María José Sosa Díaz y Jesús Valverde Berrocoso. 


\section{d) Reconocimiento de la labor de miembros de equipos directivos y coordinadores TIC}

Es importante que una misma persona no asuma dos roles dentro del centro educativo, sobre todo, cuando esa misma persona asume funciones dentro del equipo directivo. Normalmente, desarrollar la coordinación TIC es tarea ardua y que precisan de gran cantidad de tiempo para desarrollarla. Si un mismo docente asume la función de profesor a tiempo completo con la coordinación TIC, puede verse sobrecargado de trabajo y perjudicar la tarea que realiza en los cargos que ostenta (ProfPRI1, referencia 1, PRODIR, E2).

En este sentido, los entrevistados solicitan a la Administración Educativa que garantice a los equipos directivos el apoyo necesario para que puedan desarrollar de manera adecuada la función de gestión de los centros. Es necesario, por tanto, que la Administración reconozca el trabajo que realizan los coordinadores TIC, permita emplear el tiempo necesario para dicha labor, y no obligue a asumir funciones que pueden desarrollar auxiliares administrativos (DirEQD, referencia 3, PROADMIN, E2; DirEQD, referencia 3, PROADMIN, E2).

“A ver, que nos tienen que mimar un poquitín más y luego, eso. [...] yo cobro trescientos de complemento. Qué no sé. Que no lo haces por el dinero; esto lo haces porque te gusta pero que bueno, que tampoco vendría mal un poco de reconocimiento. Está claro. Los Equipos Directivos, si quieren que tengamos todas esas funciones, y cada vez vamos teniendo más funciones, y más importantes porque ha habido un tiempo que el director se le veía aquí como el que hacía los papeles, las cuentas...". (DirEQD, referencia 3, PROADMIN, E2)

\subsection{PlanificaCión Y PROYECCIÓN INSTITUCIONAL}

A continuación, se analiza el desarrollo institucional que tienen las TIC en el centro como una dimensión que da una visión del grado de integración de las tecnologías. Para ello se han analizado aspectos como la planificación y programación de las tecnologías en los centros educativos, así como aspectos de reconocimiento institucional del desarrollo de experiencias didácticas TIC, o la participación en proyectos de innovación educativos.

\subsubsection{Proyecto de integración TIC y programa curricular TIC}

Como se ha podido comprobar en el tercer estudio de investigación, actualmente todos los Proyectos Educativos de Centro, recogen un apartado de TIC. La Administración obliga a incluir esta sección en la que se deben desarrollar aspectos como las funciones del coordinador TIC, los objetivos, las actividades de formación, infraestructuras tecnológicas, la actividad formativa de informática, y las competencias básicas a desarrollar según la ley (Coor_cp02, referencia 1, PLANTIC, E3). No obstante, los PEC con los que cuentan los centros educativos, normalmente, están desactualizados. Fueron realizados hace varios años, cuando la ley lo determinaba como obligatorio. Por esta razón, a pesar de que en su día fue un documento consensuado por toda la comunidad educativa, actualmente hay docentes y miembros de la comunidad que no conocen el contenido del PEC, sobre todo, aquellos docentes interinos que han comenzado a trabajar hace poco en el centro (Coor_cp02, referencia 1, PLANTIC, E3). Además, la inclusión de las TIC en los proyectos educativos del centro es promovida

El papel de políticas educativas en los procesos de innovación e integración de las TIC en los centros educativos públicos. María José Sosa Díaz y Jesús Valverde Berrocoso. 
por un grupo de docentes interesados por el tema, por lo que en el momento en el que estos docentes cambian de centro, el apartado de tecnologías del PEC queda desfasado y en un segundo plano.

"Sí, pero el Claustro de aquel, tienen que ver bastante poco con el... El proyecto educativo, se hizo la redacción último, seis o siete años, siete me parece, por ahí. Pues me parece que desde entonces para acá, podemos quedar de los 30 que somos quedarán 10 o 12, son cosas que ahí se escriben. Luego en verdad si que ha habido bastante interés, sobre todo, suscitó bastante interés, la famosa creación de los blogs, tuvo el 'boom', y de hecho si te has metido por ahí, la página no funciona, pero blog sí que hay un montón, bastantes. Y además fue un poco un reto, que prácticamente en todos los cursos, en todos los ciclos los hubiera, hubo un 'boom', de un par de cursos que muy bien, luego ya pues la cosa se vino un poco abajo". (Dir_cp04, referencia 2, PLANTIC, E3).

En cuanto a la programación curricular adaptada a las TIC, tan sólo se ha realizado en un centro educativo de los cuatro estudiados. Los demás centros escolares no cuentan este tipo de programación, y se ajustan a lo que se establece por ley. En el caso del CP_01 se realizó una «programación vertical TIC» en la que se determinaban una sucesión de contenidos y aspectos establecidos por etapas que debían desarrollar los docentes (Dir_cp01, referencia 1, PLANTIC, E3).

Según el profesorado entrevistado, la programación curricular TIC no es utilizada actualmente en el centro, ya que no determina las competencias digitales y tratamiento de la información que se han de adquirir en cada una de las etapas educativas. Aunque, como subraya una coordinadora TIC, es importante la realización de esta programación curricular TIC pues supuso un primer paso, para concienciar sobre la importancia del uso transversal de las tecnologías en la docencia.

"Sí, hay una, pero... no está bien. Es como una secuenciación de contenidos. Es... se queda ahí. Desde Infantil hasta... hasta que salen. Entonces, eso hay que meterle mano. Pero es que hay que meter mano a tantas cosas... me refiero: no puedes meter mano a... a una programación TIC, si no metes mano a metodología, ¿eh? Porque, muy bien, a lo mejor esa... esa... esa secuenciación de contenidos me viene a mí muy bien a modo de... de guía, de control, de decir: "bueno, he cogido unos niños de $4^{\circ}$ y no... no controlan el... el... el teclado", por ejemplo, no saben todas las cosas que me ofrece el teclado. Pero si yo sigo diciendo: " $A$ ver, niños! La primera fila del teclado tiene los números!". (Coor_cp01, referencia 1, PLANTIC, E3)

Por tanto, se puede afirmar que, a pesar de los esfuerzos realizados desde la Administración para que los centros educativos incluyeran las TIC dentro de sus Proyectos Educativos de Centros, no se cuenta con un programa específico y adaptado en cada uno de los centros. Aquellos que en su día la realizaron de manera adecuada, teniendo en cuenta el consenso y las ideas del claustro, actualmente, se encuentra desfasado.

El papel de políticas educativas en los procesos de innovación e integración de las TIC en los centros educativos públicos. María José Sosa Díaz y Jesús Valverde Berrocoso. 


\subsubsection{Participación en proyectos educativos TIC y de innovación}

\section{a) Se ha participado en proyectos educativos TIC pero sin éxito}

Dos centros educativos de los que participan en la investigación (CP_03 y CP_04) señalan haber participado, bien en proyectos de convocatoria autonómica, como es el "Proyecto Atenea", o proyectos de convocatorias nacionales como la "Aldea Global". Son proyectos subvencionados por la Administración para el fomento del uso de las tecnologías en los centros. Se basaban en la realización de seminarios de formación sobre distintas herramientas TIC para la docencia, así como la creación de grupos de trabajo para la elaboración de materiales didácticos digitales (P03, referencias 3 y 4, TICSUB, E1).

Estos proyectos se ven en las intervenciones un poco difusos, los participantes no saben cómo se llaman, apenas saben explicar con claridad en qué consistía el proyecto o la subvención recibida. Lo que demuestra que la implicación de los participantes fue escasa y deja entrever que aún no existe esa labor colectiva para programar y realizar actividades TIC de forma colaborativa en el centro.

"La página web que hicimos sobre conocimiento del medio, sí fueron en estos programas de, no sé ahora como se llama, que acaban de salir ahora mismo, nos dieron un cierto dinero para realizar ese trabajo, presentamos un proyecto, nos lo aprobaron y nos dieron un dinero para elaborar ese material, material que paso a ser de ellos, de la Junta de Extremadura". (C04, referencia 1, TICSUB, E1)

Labor colectiva que sí se produce para la realización de otros proyectos de innovación educativa. Todos los centros educativos con los que se ha trabajado tienen proyectos de innovación que están trabajando algún tema en concreto. Entre ellos tenemos: Píldoras de Experiencias, Inteligencia Emocional, Biblioteca y Lectura, Competencias Básicas, Comunidades de Aprendizaje, o Escuela de Paz. Pero en ninguno de los centros se ha encontrado un proyecto de innovación que tenga como objetivo prioritario el uso de las tecnologías en el centro (P05-P06, referencia 1, TICSUB, E1). No obstante, según los docentes, en la mayoría de estos proyectos de innovación, que se han señalado, se introducen las TIC de manera transversal.

"De proyectos, de iniciativas, por ejemplo, aquí también pertenecemos a la red de centros de bibliotecas escolares, y uno de los proyectos que se están poniendo en marcha, que todavía estamos en ello, es precisamente la integración del uso de las TIC en las bibliotecas del colegio. Uno de los proyectos que tenemos en mente es comprar. Estamos ahí entre que si tablet o e-book para la biblioteca, pues para realizar actividades relacionadas con la biblioteca. Aquí tenemos un jardín botánico precioso que lo empezó a preparar un maestro, que ya está jubilado. [...] Las TIC se han metido ahí, tenemos en nuestro portal una especie de guía de nuestro jardín botánico. Luego te lo enseño también si quieres. ¿Vale? O sea, que sí, la integración de las TIC cada vez se va ramificando más en todas las actividades del centro, ¿eh? ¿Sabes? O sea que... Hemos elaborado, por ejemplo, para el día del libro, hace algunos años, un Quijote infantil, también en formato digital, una especie de... de vídeo en el que se hizo un cómic en el que los personajes eran los niños los que hablaban". (Coor_cp03, referencia 2, PROINNO, E3)

El papel de políticas educativas en los procesos de innovación e integración de las TIC en los centros educativos públicos. María José Sosa Díaz y Jesús Valverde Berrocoso.

Pág. 19 de 28 
Al analizar la cantidad de proyectos de innovación realizados en los centros, se puede afirmar que, aquellos centros que más proyectos de innovación y que más utilizan las tecnologías, son aquellos que tienen un equipo directivo innovador y que fomenta el trabajo en equipo. En estos proyectos de innovación educativa, normalmente el claustro está comprometido en la participación de las actividades (Coor_cp04, referencia 1, IMPLITIC, E3). Sin embargo, aquellos centros educativos que tienen una organización complicada debido a la idiosincrasia del centro, como los CRA, son los que menos proyectos de innovación desarrollan. La modalidad más habitual de trabajo, son los grupos de trabajo. Por lo cual, en centros educativos en los que el contacto y colaboración docentes es difícil, no se desarrolla con asiduidad este tipo de proyectos (Coor_cp02, referencia 2, PROINNO, E3).

\section{DISCUSIÓN.}

Se puede concluir que las características de la política educativa TIC y, por tanto, el modo de implantación de las tecnologías en los centros escolares, es un factor determinante a la hora de conseguir una adecuada introducción de las TIC en la educación. En este sentido, se puede afirmar que el modelo de política educativa TIC llevada a cabo influirá notablemente en la implicación del profesorado y en el uso didáctico de las herramientas tecnológicas en el aula (Alonso et al., 2010; Blignaut, Hinostroza, Els, \& Brun, 2010; Colás \& Casanova, 2010; Montero \& Gewerc, 2010; Vanderyar, 2013).

Al igual que concluyen otros estudios de investigación realizados en Extremadura, la opinión de la comunidad educativa sobre la política educativa TIC desarrollada en la región es positiva y se considera que se ha realizado una gran apuesta a nivel educativo y un gran esfuerzo económico (Sosa, Peligros, \& Díaz, 2010). Sin embargo, al igual que concluyen en investigaciones en otras regiones, se afirma que el modelo de introducción de las TIC en los centros escolares no ha sido el adecuado, ni se ha gestionado de forma coherente (Pérez, Aguaded, \& Fandos, 2010; Pérez, Hernando, \& Aguaded, 2011).

La comunidad educativa percibe que la Administración Educativa ha implantado las tecnologías en el Sistema Educativo Extremeño movida por intereses políticos y económicos. El Gobierno se ha preocupado principalmente por la instalación masiva de ordenadores, de acuerdo con su agenda política, con el objetivo de conseguir más votantes, pero sin valorar los intereses y necesidades reales de la escuela y sus docentes (Levin, 2008; Valverde, 2012). Por este motivo, de forma generalizada, se considera que no se han tomado las medidas y estrategias oportunas para favorecer el uso de los recursos tecnológicos instalados en los centros escolares o se percibe que las que se han desarrollado han sido deficientes. La política educativa TIC no está acorde con las necesidades de los docentes, ofreciéndoles aquello que no demandaron y no consideran prioritario (Montero \& Gewerc, 2010).

Así pues, la política educativa TIC extremeña, al igual que otras políticas educativas españolas, está diseñada bajo el principio «arriba-abajo» (Losada et al., 2011). Las medidas son elaboradas sin participación de los docentes en la toma de decisiones, por lo que no es un modelo adecuado para el buen desarrollo del proceso de integración de las TIC al favorecer el rechazo del profesorado, la falta de reflexión y la escasa implicación docente (J. Rodríguez, 2012; Sancho \& Correa, 2010; Vanderyar, 2013). Según los resultados obtenidos, el modelo de política TIC adecuado que debe

El papel de políticas educativas en los procesos de innovación e integración de las TIC en los centros educativos públicos. María José Sosa Díaz y Jesús Valverde Berrocoso.

Pág. 20 de 28 
desarrollar la Administración es la de centros pilotos. Con ella se instalarán los recursos tecnológicos partiendo de las necesidades e intereses de los docentes y equipos directivos de los centros. De esta forma, como también señalan Vanderlinde \& Van Braak (2011), si se escucha a los docentes se conseguirá el compromiso de todos para utilizar de forma eficiente las tecnologías implantadas en su centro.

El profesorado desconoce elementos característicos de las políticas educativas TIC (Vanderlinde \& Van Braak, 2011). No se han establecido estrategias de motivación y formación necesarias antes de la implantación de los equipos informáticos, especialmente antes de la instalación del Software Libre y plataformas de gestión y comunicación propias, lo que ha supuesto que no se aprovechen al máximo los recursos que se han instalado (Area et al., 2013). Por tanto, como se ha podido comprobar en otros estudios de investigación, es necesario que la Administración Educativa ofrezca un mayor apoyo tanto a los centros como a los profesores (García-Valcarcel \& Tejedor, 2010; Vanderyar, 2013). Este apoyo debe ser entendido como un aumento de formación especializada e información a docentes, coordinadores TIC, equipos directivos, y familias, con el objetivo de concienciar de la importancia de integración de las TIC y de capacitar en el desarrollo de sus funciones dentro de la organización educativa para el uso de las tecnologías en el centro.

El apoyo que la Administración ha desarrollado en las políticas educativas TIC llevadas a cabo, se traduce en más dotación, estableciendo medidas de mantenimiento y apoyos técnicos deficientes o insuficientes para el sostenimiento de los equipos informáticos (Montero \& Gewerc, 2010). Por este motivo, se constata que muchas infraestructuras han quedado infrautilizadas, obsoletas e inoperativas en un corto periodo de tiempo. Es necesario que la Administración Educativa instale y renueve de forma continua y progresiva los equipos tecnológicos y dote de los recursos humanos suficientes para su mantenimiento.

Para un adecuado desarrollo del proceso de integración de las TIC es necesario realizar una evaluación para determinar los cambios y mejoras que responda a las necesidades de la realidad escolar (González \& Rodríguez, 2010). Sin embargo, no se ha identificado que la Administración Educativa haya realizado un adecuado seguimiento del progreso de la integración de las tecnologías en los centros educativos. Para ello, los entrevistados instan a utilizar informes de todos los agentes educativos, como equipos directivos, los inspectores, o al mismo profesorado.

Por tanto, cualquier política TIC no puede limitarse a la dotación de materiales, equipamiento o mobiliario, entre otros aspectos técnicos (Pérez et al., 2010, 2011). Se ha comprobado que es necesario, instalar los equipos informáticos de forma escalonada, valorar las necesidades y características de los centros y los docentes, dar suficiente apoyo y formación al profesorado, los equipos directivos y las familias, así como darle un adecuado seguimiento del proceso de instauración de las TIC y mantenimiento a todos los recursos tecnológicos.

Además, según los resultados obtenidos, se recomienda que, en base a la autonomía de los centros educativos, la Administración Educativa favorezca:

- La creación de comunidades de aprendizaje específicas de tecnología para trabajar en grupo, reducir la ansiedad y falta de confianza de los docentes (Hadjithoma \& Karagiorgi, 2009).

El papel de políticas educativas en los procesos de innovación e integración de las TIC en los centros educativos públicos. María José Sosa Díaz y Jesús Valverde Berrocoso. 
- La estabilidad del profesorado en los centros en los que se lleven a cabo experiencias de integración de las tecnologías (García-Valcarcel \& Tejedor, 2010).

- El desarrollo en los centros escolares de planes de integración TIC, asesorados por expertos y tomando decisiones en base a evidencias (Tondeur, Valcke, et al., 2008; Vanderlinde \& Van Braak, 2011).

- El reconocimiento del trabajo innovación realizado por docentes, coordinadores TIC y equipo directivo (García-Valcarcel \& Tejedor, 2010).

La creación e implementación de un proyecto de integración de las TIC, o "Proyecto de Educación Digital" (PED), es un factor decisivo para una adecuada integración de las tecnologías en los centros (Gülbahar, 2007; Hew \& Brush, 2007; Valverde, 2012; Vanderlinde et al., 2014; Vanderlinde \& Van Braak, 2010).

La elaboración del proyecto supone un proceso de reflexión y análisis conjunto, a través del cual se decide los objetivos y acciones para la integración de las TIC en el centro (Gülbahar, 2007; Tondeur, Hermans, Van Braak, \& Valcke, 2008; Vanderlinde, Van Braak, \& Tondeur, 2010). De tal forma, que si toda la comunidad educativa participa en la elaboración del "Proyecto de Educación Digital" se construirá una visión compartida y se promoverá el compromiso con el desarrollo de dicho proyecto y la integración de las tecnologías en los centros (Hadjithoma \& Karagiorgi, 2009; Vanderlinde, Dexter, et al., 2012; Vanderlinde \& Van Braak, 2010, 2011).

Como se ha podido comprobar en el análisis de los "Proyecto de Educación Digital" de cada uno de los estudios de casos realizado, éstos acogen un apartado dedicado a las TIC, ya que la Administración lo establece como obligatorio. Sin embargo, la mayoría de ellos, son más bien inventarios técnicos, puesto que no contienen líneas de acción concretas y su contenido está relacionado con la organización de los recursos tecnológicos e infraestructuras (Valverde, 2012; Vanderlinde, Dexter, et al., 2012).

Además, normalmente están totalmente desactualizados, pues el claustro no conoce dicho documento y una gran parte de las personas que participaron en su elaboración ya no pertenecen a los centros educativos. Por tanto, al igual que afirman otras investigaciones como (Tilve \& Álvarez, 2009; Vanderlinde, Dexter, et al., 2012), se puede concluir que, de manera general, los centros educativos no cuentan con un adecuado "Proyecto de Educación Digital" para lograr la implementación de las TIC en los centros.

No obstante, cada centro escolar realiza un tratamiento diferente del "Proyecto de Educación Digital" que elabora. Estos proyectos responden a la idiosincrasia de los centros educativos y, sobre todo, al interés de los equipos directivos (Sánchez et al., 2011).

En la presente investigación se ha identificado un centro educativo con una dirección comprometida con la integración de las TIC, que ha promovido la creación de un PED que cuenta con una planificación curricular actualizada. Por tanto, se demuestra la importancia de la labor de los directores en dicho proceso (Vanderlinde, Van Braak, et al., 2012). Según las opiniones vertidas por los entrevistados de dicho centro educativo, en el "Proyecto de Educación Digital" es aconsejable contar con:

- Reflexión y análisis conjunto de toda la comunidad educativa.

El papel de políticas educativas en los procesos de innovación e integración de las TIC en los centros educativos públicos. María José Sosa Díaz y Jesús Valverde Berrocoso.

Pág. 22 de 28 
- Compromiso de la comunidad educativa de implementar el proyecto adecuadamente.

- Establecer objetivos, medidas y estrategias para la integración de las TIC en los centros educativos, así como contar con una programación curricular TIC(Aguaded, Pérez, \& Monescillo, 2010).

- Proyecto prioritario en el centro.

- Tener en cuenta los procesos de evaluación del propio proyecto (Vanderlinde, Dexter, et al., 2012).

Además, se afirma que para llevar a cabo un "Proyecto de Educación Digital" de forma exitosa, es necesario que este proyecto sea prioritario ante otros proyectos educativos. Los esfuerzos, tanto del equipo directivo, coordinador TIC y claustro deben estar centrados en el objetivo de integrar las TIC.

Presentación del artículo: 13 de febrero de 2017 Fecha de aprobación: 14 de marzo de 2017 Fecha de publicación: 31 de marzo de 2017

Sosa Díaz, M.J. y Valverde Berrocoso, J. (2017). El papel de políticas educativas en los procesos de innovación e integración de las TIC en los centros educativos públicos.. RED. Revista de Educación a Distancia, 53. Consultado el (dd/mm/aaaa) en http://www.um.es/ead/red/53

Esta investigación no ha recibido ninguna subvención específica de los organismos de financiación en los sectores públicos, comerciales o sin fines de lucro.

\section{REFERENCIAS}

Aesaert, K., Vanderlinde, R., Tondeur, J., \& Van Braak, J. (2013). The content of educational technology curricula: a cross-curricular state of the art. Education Tech Research Dev, 61, 131-151. https://doi.org/10.1007/s11423-012-9279-9

Aguaded, J. I., Pérez, M. amor, \& Monescillo, M. (2010). Hacia una integración curricular de las TIC en los centros educativos andaluces de primaria y secundaria. Bordón, 4, 7-23.

Aguaded, J. I., Tirado, R., \& Cabero, J. (2008). Los centros TIC en Andalucía, españa: un modelo de implementación del profesorado en la integración curricular de la tecnología. Sociotam, 18(2), 171-199.

Alí, I., Cueva, E., Fernández, M. S., Murillo, F. J., \& Gómez, M. (2004). La implantación de las tecnologías de la información y la comunicación en los centros educativos de la Comunidad de Madrid. Impacto en la docencia y en los alumnos. RELATEC: Revista Latinoamericana de Tecnología Educativa, 3. Retrieved from http://campusvirtual.unex.es/revistas/index.php?journal=relatec\&page=article\&op $=$ viewArticle $\&$ path $\% 5 \mathrm{~B} \% 5 \mathrm{D}=151$

El papel de políticas educativas en los procesos de innovación e integración de las TIC en los centros educativos públicos. María José Sosa Díaz y Jesús Valverde Berrocoso. 
Alonso, C., Casablancas, S., Domingo, L., Guitert, M., Moltó, Ó., Sánchez, J.-A., \& Sancho, J. (2010). De las propuestas de la Administración a las prácticas del aula. Revista de educación, 352, 53-76.

Area, M. (2002). Integración escolar de las nuevas tecnologías. Entre el deseo y la realidad. Organización y gestión escolar: Revista del Forum Europeo de Administradores de la Educación, 10(6), 14-18.

Area, M. (2005). Tecnologías de la información y comunicación en el sistema escolar. Una revisión de las líneas de investigación. Relieve, 11(1), 3-25.

Area, M. (2008). Una breve historia de las políticas de incorporación de las tecnologías digitales al sistema escolar en España. Quaderns Digitals, 51. Retrieved from http://www.quadernsdigitals.net/index.php?accionMenu=hemeroteca.VisualizaArti culoIU.visualiza\&articulo_id=10454

Area, M., Sanabria, A., \& Vega, A. M. (2013). Las políticas educativas TIC (Escuela 2.0) en las Comunidades Autónomas de España desde la visión del profesorado. Revista científica de Tecnología Educativa, 3(1), 74-88.

Baylor, A. L., \& Ritchie, D. (2002). What factors facilitate teacher skill, teacher morale, and perceived student learning in technology-using classrooms? Computers \& Education, 39(4), 395-414. https://doi.org/http://dx.doi.org/10.1016/S0360-1315 (02) 00075-1

Blignaut, A. S., Hinostroza, J. E., Els, C. J., \& Brun, M. (2010). ICT in education policy and practice in developing countries: South Africa and Chile compared through SITES 2006. Computers \& Education, 55, 1552-1563. https://doi.org/10.1016/j.compedu.2010.06.021

Colás, P., \& Casanova, J. (2010). Variables docentes y de centro que generan buenas prácticas con TIC. Revista teoría de la educación. Educación y cultura en la Sociedad de la Información, 11(3), 121-147.

Correa, J. M., \& Martínez, A. (2010). ¿Qué hacen las escuelas innovadoras con la tecnología?: las TIC al servicio de la escuela y la comunidad en el colegio Amara Berri. Revista teoría de la educación. Educación y cultura en la Sociedad de la Información, 11(3), 230-261.

De Pablos, P., Colás, P., \& González, T. (2010). Factores facilitadores de la innovación con TIC en los centros escolares. Un análisis comparativo entre diferentes políticas educativas autonómicas. Revista de educación, 352, 23-53.

De Pablos, P., Colás, P., \& Villaciervos, P. (2010). Políticas educativas, buenas prácticas y TIC en la Comunidad Autónoma Andaluza. Revista teoría de la educación. Educación y cultura en la Sociedad de la Información, 11(1), 180-202.

Escontrela, R., \& Stojanovi, L. (2004). La integración de las TIC en la educación: apuntes para un modelo pedagógico pertinente. Revista de pedagogía, 25(74). Retrieved from http://www.scielo.org.ve/scielo.php?pid=s079897922004000300006\&script=sci_arttext

Espuny, C., Gisbert, M., \& Coiduras, J. (2010). La dinamización de las TIC en las escuelas. Edutec: Revista electrónica de tecnología educativa, 32, 1-16.

El papel de políticas educativas en los procesos de innovación e integración de las TIC en los centros educativos públicos. María José Sosa Díaz y Jesús Valverde Berrocoso.

Pág. 24 de 28 
Fishman, B., \& Zhang, B. (2003). Planning for technology: The link between intentions and use. Educational Technology, 43, 14-18.

García-Valcarcel, A., \& Tejedor, F. J. (2010). Evaluación de procesos de innovación escolar basados en el uso de las TIC desarrollados en la Comunidad de Catilla y León. Revista de educación, 352, 125-147.

Gargallo, B., Suárez, J., Morant, F., Marín, J. M., Martínez, M., \& Díaz, I. (2004). Un primer diagnóstico del uso de internet en los centros escolares de la Comunidad Valenciana. Procesos de formación y efectos sobre la calidad de la educación. Valencia: IVECE. Retrieved from http://www.n.mec.es/cide/espanol/publicaciones/investigacion/estudios/inv2004tic /02.pdf

Glaser, B., \& Strauss, A. (1967). The Discovery of Grounded Theory: strategies for qualitative research. New York: Aldine de Gruyter.

González, T., \& Rodríguez, M. (2010). Valor añadido de las buenas prácticas con TIC en los centros educativos. Revista teoría de la educación. Educación y cultura en la Sociedad de la Información, 11(3), 262-282.

Gülbahar, Y. (2007). Technology planning: A roadmap to successful technology integration in schools. Computers \& Education, 49, 943-956. https://doi.org/10.1016/j.compedu.2005.12.002

Hadjithoma, C., \& Karagiorgi, Y. (2009). The use of ICT in primary schools within emerging communities of implementation. Computers \& Education, 52, 83-91. https://doi.org/10.1016/j.compedu.2008.06.010

Hew, K. F., \& Brush, T. (2007). Integrating technology into K-12 teaching and learning: current knowledge gaps and recommendations for future research. Educational Technology Research and Development, 55, 223-252. https://doi.org/http://dx.doi.org/10.1007/s11423-006-9022-5

Kopcha, T. J. (2012). Teachers' perceptions of the barriers to technology integration and practices with technology under situated professional development. Computers \& Education, 50, 1109-1121. https://doi.org/10.1016/j.compedu.2012.05.014

Lázaro, J. L., \& Gisbert, M. (2007). La integración de las TIC en los centros escolares de educación infantil y primaria: condiciones previas. Pixel-bit. Revista de Medios y Educación, 28, 27-34.

Levin, B. (2008). How governments decide: The role of research. In R. Desjardins \& K. Rubensen, Research of policy and research for policy in an era of transnational education policy making. (pp. 44-57). Aarhaus: Aarhaus University Press.

Losada, D., Karrera, I., \& Correa, J. M. (2011). Políticas sobre la integración de las TIC en la escuela de la Comunidad Autonoma del País Vasco. RELATEC: Revista Latinoamericana de Tecnología Educativa, 10(1), 21-35.

Martínez, M. E. (2006). Políticas autonómicas para la integración de las TIC en centros educativos. RELATEC: Revista Latinoamericana de Tecnología Educativa, 5(2), 97-112.

El papel de políticas educativas en los procesos de innovación e integración de las TIC en los centros educativos públicos. María José Sosa Díaz y Jesús Valverde Berrocoso. 
Mioduser, D., Nachmias, R., Tubin, D., \& Forkosh-Baruch, A. (2003). Analysis Schema for the Study of Domains and Levels of Pedagogical Innovation in Schools Using ICT. Education and Information Technologies, 8(1), 23-26.

Montero, L., \& Gewerc, A. (2010). De la innovación deseada a la innovación posible. Escuelas Alteradas por las TIC. Revista de currículum y formación del profesorado, 14(1), 303-318.

Nachmias, R., Mioduser, D., Cohen, A., Tubin, D., \& Forkosh-Baruch, A. (2004). Factors involver in the implementation of pedagogical Innovations Using technology. Education and Information Technologies, 9(3), 291-308.

Pérez, M. A., Aguaded, J. I., \& Fandos, I. (2010). Percepciones, retos y demandas de los directores y coordinadores de los centros TIC andaluces. Siglo XXI, 28(1). Retrieved from http://rabida.uhu.es/dspace/bitstream/handle/10272/6368/Percepciones\%2c\%20ret os $\% 20 \mathrm{y} \% 20$ demandas.pdf? sequence $=2$

Pérez, M. A., Aguaded, J. I., \& Fandos, M. (2009). Una política acertada y la formación permanente del profesorado, claves en el impulso de los centros TIC de andalucía. Edutec: Revista electrónica de tecnología educativa, 29, 1-17.

Pérez, M. A., Hernando, Á., \& Aguaded, J. I. (2011). La integración de las TIC en los centros educativos: percepciones de los coordinadores y directores. Estudios Pedagogicos, 307(2), 197-211.

Protheroe, N. (2005). Technology and student achievement. National Association of Elementary School Principals, 82(2), 46-48.

Rodríguez, G., Gil, J., \& García, E. (1999). Metodología de la Investigación Cualitativa. Malaga: Algibe.

Rodríguez, J. (2012). Análisis sobre la integración en el sistema educativo de las TIC: proyectos institucionales y formación permanente. Revista teoría de la educación. Educación y cultura en la Sociedad de la Información, 13(3), 129-144.

Sanabria, A. (2006). Las TIC en el sistema escolar de Canarias: Los programas institucionales de innovación educativa para la integración curricular de las Tecnologías de la Información y Comunicación. RELATEC: Revista Latinoamericana de Tecnología Educativa, 5(2), 191-202.

Sánchez, E., Sánchez, R., \& Ruiz, J. (2011). Estudio sobre la influencia del género en la incorporación de las TIC a los centros educativos. 2011, 12(2), 54-80.

Sancho, J., \& Correa, J. M. (2010). Cambio y continuidad en sistemas educativos en transformación. Revista de educación, 352, 17-21.

Sanz, M. D., Martínez, E., \& Pernas, E. (2010). Innovación con TIC y cambio sostenible. Un proyecto de investigación colaborativa. Revista de currículum y formación del profesorado, 14(1), 319-337.

Sosa, M. J. (2013). 30 años de Políticas Educativas TIC en Portugal. REDEX - Revista de Educación de Extremadura, 3(5), 83-98.

El papel de políticas educativas en los procesos de innovación e integración de las TIC en los centros educativos públicos. María José Sosa Díaz y Jesús Valverde Berrocoso. 
Sosa, M. J., Peligros, S., \& Díaz, D. (2010). Buenas Prácticas organizativas para la integración de las TIC en el sistema educativo extremeño. Revista teoría de la educación. Educación y cultura en la Sociedad de la Información, 11(3), 148-179.

Strauss, A., \& Corbin, J. (2002). Bases de la investigación cualitativa. Técnicas y procedimientos para desarrollar la teoría fundamentada. Colombia: Universidad de Antioquia.

Tilve, M. D., \& Álvarez, Q. (2009). Un estudio de caso sobre un proyecto de innovación con TIC en un centro educativo de Galicia: ¿Acción o reflexión? Bordón, 61(1), 95-108.

Tilve, M. D., Gewerc, A., \& Álvarez, Q. (2009). Proyectos de innovación curricular mediados por TIC: $\quad$ Un estudio de caso. RELATEC: Revista Latinoamericana de Tecnología Educativa, 8(1), 65-81.

Tondeur, J., Hermans, R., Van Braak, J., \& Valcke, M. (2008). Exploring the link between teachers' educational belief profiles and different types of computer use in the classroom. 24, 6, 2541-2553. https://doi.org/http://dx.doi.org/10.1016/j.chb.2008.02.020

Tondeur, J., Valcke, M., \& Van Braak, J. (2008). A multidimensional approach to determinants of computer use in primary education: teacher and school characteristics. Journal of Computer Assisted Learning, 24(6), 494-506. https://doi.org/10.1111/j.1365-2729.2008.00285.x

Tondeur, J., Van Keer, H., Van Braak, J., \& Valcke, M. (2008). ICT integration in the classroom: Challenging the potential of a school policy. Computers \& Education, 51, 212-223. https://doi.org/10.1016/j.compedu.2007.05.003

Valverde, J. (2012). Políticas educativas en tecnología educativa: el papel de la investigación y la autonomía del centro en la toma de decisiones. Revista científica de Tecnología Educativa, 1(1), 43-50.

Vanderlinde, R., Aesaert, K., \& Van Braak, J. (2014). Institutionalised ICT use in primary education: A multilevel analysis. Computers \& Education, 72, 1-10. https://doi.org/dx.doi.org/10.1016/j.compedu.2013.10.007

Vanderlinde, R., Dexter, S., \& Van Braak, J. (2012). School-based ICT policy plans in primary education: elements, typologies and underlying processes. British Journal of Educational Technology, 43(3), 505-519. https://doi.org/doi:10.1111/j.14678535.2011.01191.x

Vanderlinde, R., \& Van Braak, J. (2010). The e-capacity of primary schools: Development of a conceptual model and scale construction from a school improvement perspective. Computers \& Education, 55, 541-553. https://doi.org/10.1016/j.compedu.2010.02.016

Vanderlinde, R., \& Van Braak, J. (2011). A new ICT curriculum for primary education in Flanders: defining and predicting teachers' perceptions of innovation attributes. Educational Technology \& Society, 14(2), 124-135.

Vanderlinde, R., Van Braak, J., \& Dexter, S. (2012). ICT policy planning in a context of curriculum reform: Disentanglement of ICT policy domains and artifacts.

$\overline{\text { El papel de políticas educativas en los procesos de innovación e integración de las TIC en los centros }}$ educativos públicos. María José Sosa Díaz y Jesús Valverde Berrocoso.

Pág. 27 de 28 
$\begin{array}{llll}\text { Computers } \quad \& \quad \text { Education, } & \text { 1339-1350. }\end{array}$ https://doi.org/10.1016/j.compedu.2011.12.007

Vanderlinde, R., Van Braak, J., \& Hermans, R. (2009). Educational technology on a turning point: curriculum implementation in Flanders and challenges for schools. Education Tech Research Dev, 57, 573-548. https://doi.org/10.1007/s11423-0099126-9

Vanderlinde, R., Van Braak, J., \& Tondeur, J. (2010). Using an online tool to support school-based ICT policy planning in primary educationjcal_358. Journal of Computer Assisted Learning, 26, 434-447. https://doi.org/10.1111/j.13652729.2010.00358.x

Vanderlinde, R., Van Braak, J., Windt, V. D., Tondeur, J., Hermans, R., \& Sinnaeve, I. (2008). Technology curriculum and planning for technology in schools: The Flemish case. TechTrends, 52(2), 23-26.

Vanderyar, T. (2013). Practice as policy in ICT for education: Catalysing communities of practice in education in South Africa. Technology in Society, En prensa.

Vieira, E. A., \& Silva, R. M. G. (2009). Tecnologias no cotidiano escolar: limites e possibilidades. RELATEC: Revista Latinoamericana de Tecnología Educativa, 8(2), 109-125.

El papel de políticas educativas en los procesos de innovación e integración de las TIC en los centros educativos públicos. María José Sosa Díaz y Jesús Valverde Berrocoso. 